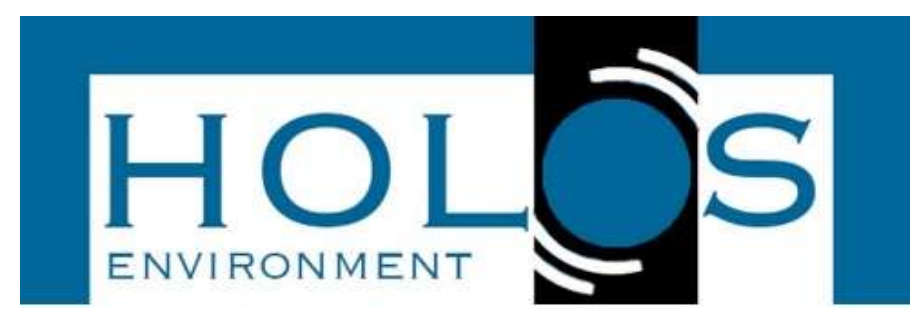

\title{
ABORDAGEM MULTICRITÉRIO NA SELECÃO DE ALTERNATIVAS DE TRATAMENTO DE EFLUENTES: UMA REVISẨO SISTÊMICA DA LITERATURA
}

\author{
MULTICRITERIA APPROACH IN THE SELECTION OF WASTEWATER TREATMENT \\ ALTERNATIVES: A SYSTEMATIC LITERATURE REVIEW
}

\author{
Andreia dos Santos Goffi ${ }^{1}$, Sandro César Bortoluzzi ${ }^{1}$, Flavio Trojan ${ }^{1}$, Andressa Goffi ${ }^{1}$
}

Artigo recebido em: 19/09/2017 e aceito para publicação em: 19/01/2018.

DOI: http://dx.doi.org/10.14295/holos.v18i1.12246

Resumo: O estudo bibliográfico estruturado tem se tornado uma excelente ferramenta para avaliar tendências cientificas em diferentes campos do conhecimento. Neste sentido, este trabalho busca analisar as oportunidades de pesquisa, apresentando uma visão geral a cerca da tomada de decisão em projetos de tratamento de efluentes no cenário internacional. ProKnow-C (Knowledge Development Process Constructivist) foi a ferramenta de intervenção utilizada, que consiste em um processo estruturado para geração de conhecimento em um tema determinado a partir dos interesses e limitações do pesquisador. Ao final do processo de busca foram identificados 16 artigos com reconhecimento científico e conteúdo alinhado ao tema de pesquisa. Os primeiros resultados da pesquisa demostraram uma acentuada tendência de crescimento a cerca do tema nos últimos 7 anos. A análise aprofundada dos trabalhos demonstrou que a construção dos modelos no cenário internacional segue padrões possíveis de serem replicados em diferentes cenários, considerando diferentes aspectos e necessidades dos decisores. De modo geral, os estudos aplicando métodos de apoio à decisão, especialmente para a seleção de tecnologias de tratamento de efluentes se demonstraram de alto impacto, e por consequência alta relevância.

Palavras-chave: Bibliometria. Tomada de decisão. Tratamento de Efluentes. Análise sistêmica da literatura.

Abstract: The structured bibliographic study has become an excellent tool to evaluate scientific trends in different fields of knowledge. In this sense, this work aims to analyse the research opportunities, by presenting an overview about decision making in sanitation projects in the international scenario. ProKnow-C (Knowledge Development Process - Constructivist) was the intervention tool applied in this work, which consists of a structured process for generating knowledge over a given topic based on the researcher interests and limitations. At the end of the search process, 16 articles with scientific recognition and content aligned with the research were identified. The first survey results have showed a increase growth trend around the theme in the last 7 years. The in-depth analysis have showed that the construction of the international models follows standard methodologies which can be replicated in different scenarios, by considering different aspects and decision makers preferences. Studies applying decision support methods in the sanitary area, especially for the selection of wastewater treatment technologies have been shown to have a high impact, and consequently a high relevance.

Keywords: Bibliometrics. Decision taking. Wastewater treatment. Systematic literature review

\footnotetext{
1 Universidade Tecnológica Federal do Paraná. Emails: (andreiagoffi@gmail.com,sandro@utfpr.edu.br, trojan@utfpr.edu.br, goffiandressa@gmail.com)
} 


\section{INTRODUÇÃO}

As contaminações dos corpos d'água representam sérios riscos à saúde pública, sendo estreita a relação entre a qualidade de água e as enfermidades que acometem as populações, especialmente aquelas não atendidas por serviços de saneamento. Assim sendo, a escolha de um adequado sistema de tratamento de esgotos é de vital importância para a saúde pública e para o meio ambiente, porque evita riscos de contaminação, transmissão de doenças, problemas estéticos, recreacionais, maus odores, mortandade de peixes, problemas com a toxicidade das algas, entre outros (METCALF, EDDY, 2003; LIBÂNIO, 2005; SPERLING, 2014).

Contudo, a seleção de um sistema de tratamento de esgoto é, de modo geral, bastante complexa, uma vez que esta envolve uma série de alternativas e variáveis, tanto sociais, econômicas, ambientais e técnicas (ZENG et al., 2007; FLORES et al., 2008; HAKANEN et al., 2011; MOLINOS et al., 2015).

Frente tamanha complexidade alguns estudos têm sido realizados com o objetivo de reduzir incertezas, por meio da aplicação de técnicas e métodos de apoio a decisão. Neste sentido, ferramentas multicritério são em geral bastante úteis, uma vez que auxiliam na estruturação do problema de forma clara e sistemática (KARIMI et al., 2011; MAURER et al., 2012). Especialmente, quanto à resolução de problemas envolve um grande número de variáveis, as quais por muitas vezes são conflitantes entre si (HAKANEN et al., 2011).

Sendo assim, o conhecimento a respeito do tema é de grande relevância, uma vez que contribuirá com cenário brasileiro de seleção de tecnologias aplicadas ao tratamento de efluentes domésticos, o qual ainda é bastante rudimentar (SPERLING, CHERNICHARO, 2002; HUNT, 2013). Segundo Zhang et al. (2015) a pesquisa bibliográfica é aplicada em vários campos da ciência para avaliar as tendências de pesquisa, através da investigação das características de diferentes publicações. Deste modo, o levantamento bibliográfico quando realizado de forma estruturada, seguindo um conjunto ordenado de procedimentos, fornece uma base robusta de informações a cerca do tema (LIMA, MIOTO, 2007).

Com base nisto, este trabalho tem como objetivo principal identificar as oportunidades de pesquisa, apresentando uma visão geral acerca da tomada de decisão em projetos de saneamento no cenário internacional. Tendo como objetivos específicos: (i) selecionar um portfólio bibliográfico com trabalhos que utilizam ferramentas auxiliares no processo de tomada de decisão na área sanitária; (ii) realizar análise bibliométrica do Portifólio Bibliográfico e de suas referências para evidenciar os principais periódicos, 
artigos, autores e palavras-chave de maior destaque; (iii) analisar o Portfólio Bibliográfico sob lentes da visão construtivista.

\section{METODOLOGIA}

Os métodos de pesquisa podem ser classificados quanto à forma de abordagem, em qualitativos e quantitativos. O enfoque qualitativo procura coletar dados sem a necessidade de medição numérica para descobrir ou aperfeiçoar as questões de pesquisa. $O$ enfoque quantitativo faz uso da coleta de dados para testar hipóteses por meio de medição numérica e análise estatística com a finalidade de estabelecerem padrões de comportamento (SAMPIERI; COLLADO; LUCIO, 2006; CAUCHICK et al., 2012). Com base nisto, esta pesquisa apresenta uma abordagem combinada das técnicas qualitativa e quantitativa, pois tem por objetivo levantar e detalhar o fenômeno, utilizando-se de ferramentas matemáticas, e da pesquisa bibliográfica estruturada.

A ferramenta de intervenção utilizada foi ProKnow-C (Knowledge Development Process - Constructivist) que consiste em um processo estruturado para a geração de conhecimento sobre um determinado tema a partir dos interesses e limitações do pesquisador, seguindo uma visão construtivista e exigindo uma interação constante entre o pesquisador e o objeto de sua pesquisa (BORTOLUZZI et al., 2011; ENSSLIN et al., 2012; ENSSLIN et al., 2014; LACERDA, ENSSLIN, 2014; DUTRA et al., 2015a; DUTRA et al., 2015b; ENSSLIN et al., 2015).

Este processo metodológico consiste basicamente em 5 etapas. Na primeira etapa, as palavras-chave e os bancos de dados são selecionados. Na segunda etapa, os filtros são elaborados, tais como trabalhos duplicados, período de pesquisa, fator de impacto entre outros. Na terceira etapa, todos os títulos são lidos para conduzir a exclusão dos que não são adequados ao campo de pesquisa. O quarto passo é a leitura completa dos resumos. E, finalmente, no quinto passo é realizada a leitura completa do trabalho. Todas as etapas são mostradas na Figura 1.

A fim de conhecer melhor as características dos trabalhos foi realizada a análise sistêmica do portfólio bibliográfico. Para isso, buscou-se evidenciar os destaques e as oportunidades (carências) de conhecimentos encontrados na amostra estudada através da aplicação das lentes de pesquisa, adaptadas de Ensslin et al., (2010), conforme apresentado na Figura 2. 
Figura 1 - Abordagem da revisão da literatura

\begin{tabular}{|c|c|c|c|c|}
\hline $1^{\circ}$ & & & & (3083 Artigos) \\
\hline \multirow{8}{*}{$\begin{array}{l}\text { Palavras-Chave } \\
\text { - Multicriteria } \\
\text { - Validation } \\
\text { - Selection } \\
\text { - Sewage } \\
\text { - Sanitation } \\
\text { - Wastewater } \\
\text { - Multiple } \\
\text { objectives } \\
\text { Banco de Dados } \\
\text { - Web of Science } \\
\text { - Scopus } \\
\text { - Science Direct }\end{array}$} & $2^{\circ}$ & & \multicolumn{2}{|r|}{ (2015 Artigos) } \\
\hline & Duplicados & $3^{\circ}$ & & (205 Artigos) \\
\hline & & \multirow{6}{*}{ Leitura de títulos } & $4^{\circ}$ & (56 Artigos) \\
\hline & - Até 2016 & & \multirow{5}{*}{ Leitura de resumos } & $5^{\circ} \quad(16$ Artigos $)$ \\
\hline & Apenas artigos & & & \multirow{4}{*}{$\begin{array}{l}\text { Leitura integral do } \\
\text { artigo }\end{array}$} \\
\hline & Número de & & & \\
\hline & citaçōes & & & \\
\hline & $\begin{array}{l}-6 \text { ou 1 para } \\
\text { trabalhos } \\
\text { publicados em } \\
2016\end{array}$ & & & \\
\hline
\end{tabular}

Figura 2 - Lentes de pesquisa

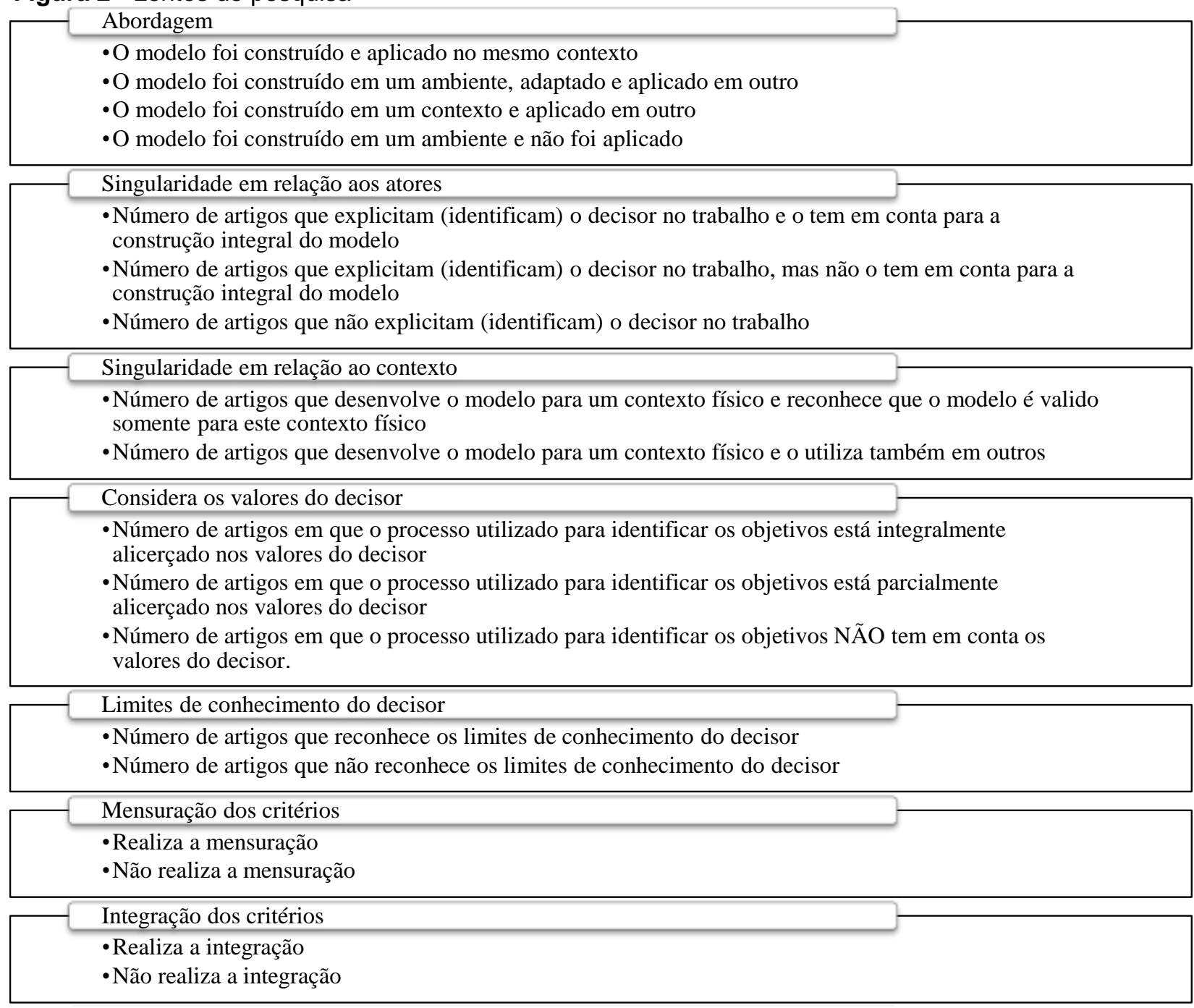




\section{RESULTADOS E DISCUSSÃO}

A análise bibliométrica é uma ferramenta utilizada para quantitativamente e estatisticamente conduzir a análise de conteúdo, a fim de destacar possíveis padrões de publicações científicas dentro de um determinado tema (HO, 2008; ZHI, GUODONG, 2012). Além disso, a partir dos métodos bibliométricos é possível avaliar as tendências de desenvolvimento ou orientações para futuras pesquisas ( $\mathrm{LI}$ et al., 2009; ZHI, GUODONG, 2012). Baseado nisto, este tópico busca demonstrar e analisar os principais autores, periódicos e trabalhos publicados sob a temática do processo decisório no saneamento.

Com base neste primeiro conjunto de dados levantados foi elaborado um gráfico a fim de conhecer a distribuição de publicações relacionadas ao tema saneamento utilizando métodos multicritérios ao longo dos anos que a pesquisa abrangeu (Figura 3).

Figura 3 - Número de artigos publicados entre 1970-2016

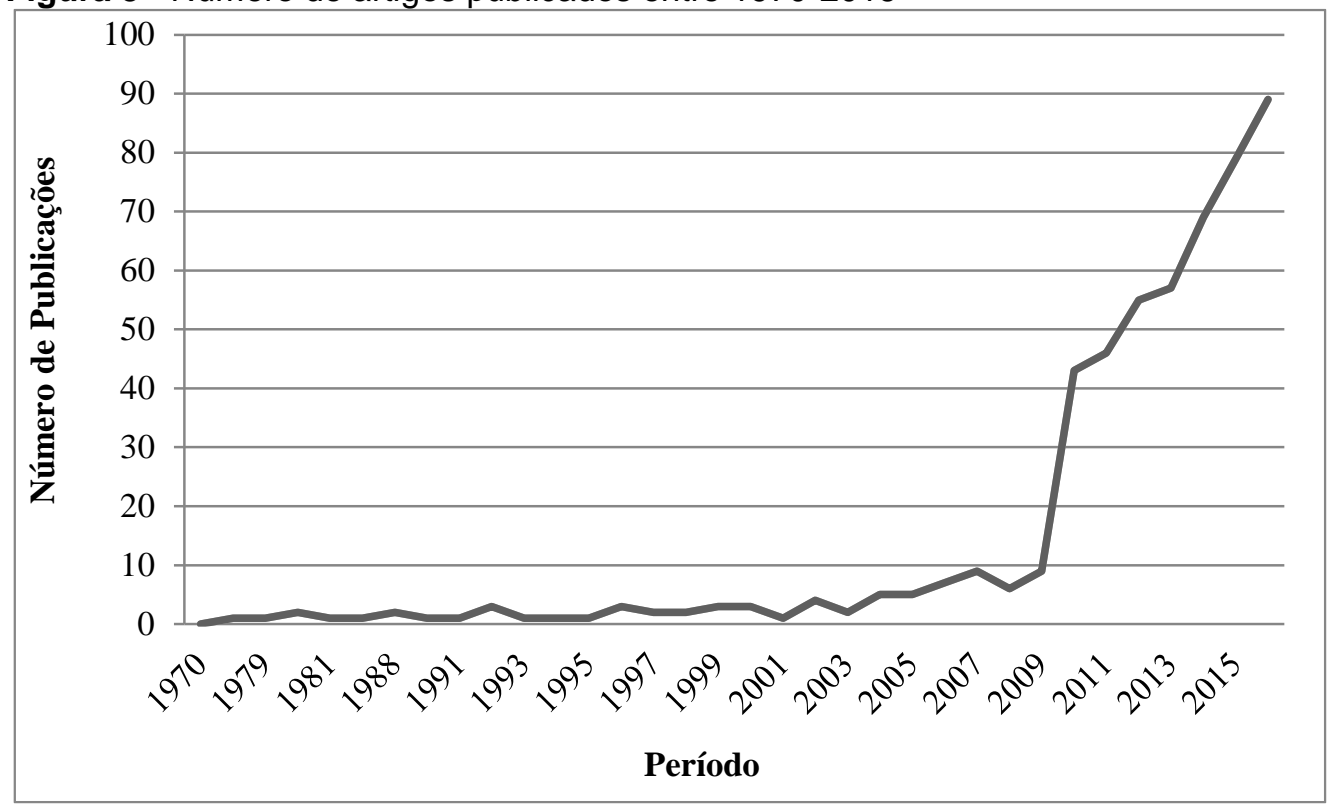

De acordo com a Figura 3 é possível notar uma tendência ascendente com um aumento significativo na taxa de crescimento marginal durante os últimos cinco anos. Onde mais de $81 \%$ dos artigos foram publicados alinhados ao tema saneamento e aplicação de métodos de apoio à tomada de decisão. Período no qual, praticamente todos os artigos com aplicações diretas a seleção de tecnologias de tratamento de efluentes foram encontrados.

O que abre espaço para o seguinte questionamento: como é garantida a qualidade das decisões no âmbito sanitário, considerando o limitado número de estudos a cerca do 
tema. Decisões geridas de forma precária e pouco estruturadas resultam em baixa qualidade e alto impacto na eficiência e custos do tratamento, o que por consequência gera pressão ambiental e econômica principalmente em países em desenvolvimento (TSAGARAKIS et al., 2002; KALBAR et al., 2012; SPERLING, 2014). Fato este que robustece a necessidade e a oportunidade de pesquisa neste segmento, pois poucos são os estudos conduzidos neste sentido, tendo em vista tamanha relevância deste tema, especialmente no cenário atual.

O portfólio bibliográfico (PB) foi definido após a leitura integral dos artigos selecionados, os quais estavam disponíveis de forma gratuita no portal CAPES. Realizada a leitura dos 56 artigos disponíveis na íntegra chegou-se a um total de 16 artigos alinhados com os objetivos da pesquisa, estes que constituíram o portfólio bibliográfico (Tabela 1).

Tabela 1 - Portfólio Bibliográfico

\begin{tabular}{lc}
\hline & Referências \\
\hline Refsgaard, (2006) & Kalbar et al. (2012a) \\
Zeng et al. (2007) & Kalbar et al. (2012b) \\
Flores et al. (2008) & Garrido et al. (2012) \\
Vasiloglou et al. (2009) & Maurer et al. (2012) \\
Karimi et al. (2011) & Kalbar et al. (2013) \\
Rashid et al (2011) & Garrido et al. (2014) \\
Sala et al. (2011) & Molinos et al. (2014) \\
Molinos et al. (2012) & Molinos et al. (2015) \\
\hline
\end{tabular}

Dentre os 16 artigos do portfólio bibliográfico, 6 (37,5\%) foram publicados pelo Journal of Environmental Management e $3(18,7 \%)$ pelo periódico Science of The Total Environment o que demonstra o alto grau de interesse destes periódicos a aplicações de métodos e ferramentas auxiliares no processo de tomada de decisão quando aplicados ao saneamento. É importante ressaltar, o alto fator do impacto de todos os periódicos ao qual o tema foi submetido e aceito para publicação, o que ressalta a importância e relevância dos estudos aplicados ao tema, conforme pode ser observado na Tabela 2. 
Tabela 2 - Portfólio Bibliográfico

\begin{tabular}{|c|c|c|c|c|}
\hline Periódicos & $\begin{array}{c}N^{\circ} \text { de } \\
\text { freq. no } \\
\text { P.B }\end{array}$ & $\begin{array}{l}\text { Freq. } \\
\text { relativa } \\
(\%)\end{array}$ & $\begin{array}{c}\text { Freq. } \\
\text { Acumulada } \\
(\%)\end{array}$ & $\begin{array}{l}\text { Fator de } \\
\text { Impacto }\end{array}$ \\
\hline Journal of Environmental Management & 6 & 38 & 38 & 4,010 \\
\hline Science of The Total Environment & 3 & 19 & 56 & 4,900 \\
\hline Desalination & 1 & 6 & 63 & 5,527 \\
\hline Environmental Modelling \& Software & 1 & 6 & 69 & 4,420 \\
\hline $\begin{array}{l}\text { International Journal of Environmental } \\
\text { Science and Technology }\end{array}$ & 1 & 6 & 75 & 2,190 \\
\hline Technology in Society & 1 & 6 & 81 & 1,039 \\
\hline Water Resources & 1 & 6 & 88 & 0,388 \\
\hline $\begin{array}{l}\text { Environment and Planning C: } \\
\text { Government and Policy }\end{array}$ & 1 & 6 & 94 & 2,161 \\
\hline Chemical Engineering Journal & 1 & 6 & 100 & 6,159 \\
\hline
\end{tabular}

${ }^{*}$ Fator de impacto retirado de Elsevier (2017) e Springer (2017).

Foi possível notar, de acordo com a Tabela 2 que apenas uma pequena percentagem dos periódicos analisados é específica da área de tratamento de efluentes e águas, ao passo que muitos artigos foram publicados em uma ampla variedade de revistas acadêmicas. Uma possível explicação é que os estudos relacionados à tomada de decisão no âmbito sanitário são estudos geralmente interdisciplinares, portanto esses se adequam a diferentes revistas, pois essas normalmente têm diversas áreas e escopos.

Quanto aos artigos, verificou-se que o artigo Optimization of wastewater treatment alternative selection by hierarchy grey relational analysis elaborado pelos autores Guangming Zenga, Ru Jianga, Guohe Huanga, Min Xua e Jianbing Lia, demonstrou ser de elevado destaque visto que este teve o maior número de citações, representado mais de $35 \%$ quando comparado com os demais artigos (165 citações de acordo com o Google Acadêmico no período de setembro de 2017).

Em relação à análise referente aos autores foi possível verificar que María Molinos Senante é a autora que mais se destacou entre os demais (10\%), seguido por France Sancho Hernandez (6\%), sendo que esses autores possuem 3 dos artigos em comum, o que pode indicar um possível grupo de pesquisa alinhado ao tema.

Quanto à análise das palavras-chave constatou-se que a maioria delas estava relacionada ao terceiro eixo de pesquisa Wastewater treatment com representatividade de cerca de $20 \%$, seguido pelo segundo eixo de pesquisa Multicriteria methods com 15,28\%, 
e por fim o terceiro eixo Decision Support system com 15,28\%. As demais palavras-chave totalizaram aproximadamente $52 \%$, contudo esse alto valor se justifica observado que estes artigos são estudos diferentes que apresentam palavras-chave específicas a fim de descrever características como: localidade, método de estudo, abrangência.

A metodologia proposta por Ensslin et al. (2010) sugere a análise das características das referências dos artigos do Portfólio. Portanto, nesta fase foram avaliadas 385 referências, ou seja, 385 trabalhos os quais foram publicados em 131 periódicos. Deste modo, foram identificadas 9 revistas como sendo as principais responsáveis pelas publicações dos trabalhos referenciados pelo portfólio, cerca de 50\% dos 385 artigos. No qual o periódico Water Science Technology ficou em primeiro lugar no número de artigos publicados nas referências do portfólio bibliográfico (62, 16\%), seguido pelo periódico Journal of Environmental Management (30, 8\%), e da revista European Journal of Operational Research (25, 6\%).

Segundo Dultra et al. (2015) a análise sistêmica é uma etapa é bastante importante e também delicada, pois exige uma afiliação teórica, pela qual irão resultar as percepções e valores diante do contexto analisado, portanto diante dos aspectos da visão construtivista os resultados observados quanto a aplicação das lentes foram analisados. A Figura 4 apresenta os resultados da análise de sistêmica dos artigos selecionados, segundo cada lente de avaliação.

A Figura 4 (a) apresenta o levantamento das principais bases de construção dos modelos. Ao passo que alguns trabalhos utilizam apenas os especialistas ou o decisor (tal como prefeito, gestor de projetos ou outro cargo específico) outros consideram ambos os aspectos, além do contexto de aplicação. O conhecimento a respeito desses agentes é de grande relevância, uma vez que isso garante que os modelos sejam construídos de maneira sólida e estruturada.

A lente abordagem, Figura 4 (b), tem por objetivo verificar a harmonia da origem dos dados para construção do modelo com sua efetiva aplicação. Foi possível observar por meio desta lente que $88 \%$ dos modelos de seleção de tecnologias de tratamento de efluentes foram construídos com base na literatura, especialistas e legislação e então aplicados a contextos específicos. Essa constatação chama atenção para o pequeno número de trabalhos que tem em vista aspectos específicos do cenário. Apontando para a necessidade de estruturação de contextos decisórios menos generalistas, de forma a propor soluções para os problemas de seleção de maneira a identificar as particularidades de cada contexto. 
Figura 4 - Lentes de pesquisa: quanto à origem dos dados para a construção do modelo (a); quanto à abordagem (b); quanto à singularidade em relação aos atores (c); quanto à singularidade em relação aos contextos (d); quanto os valores do decisor (e); quanto à mensuração dos critérios (f); quanto à integração dos critérios $(\mathrm{g})$

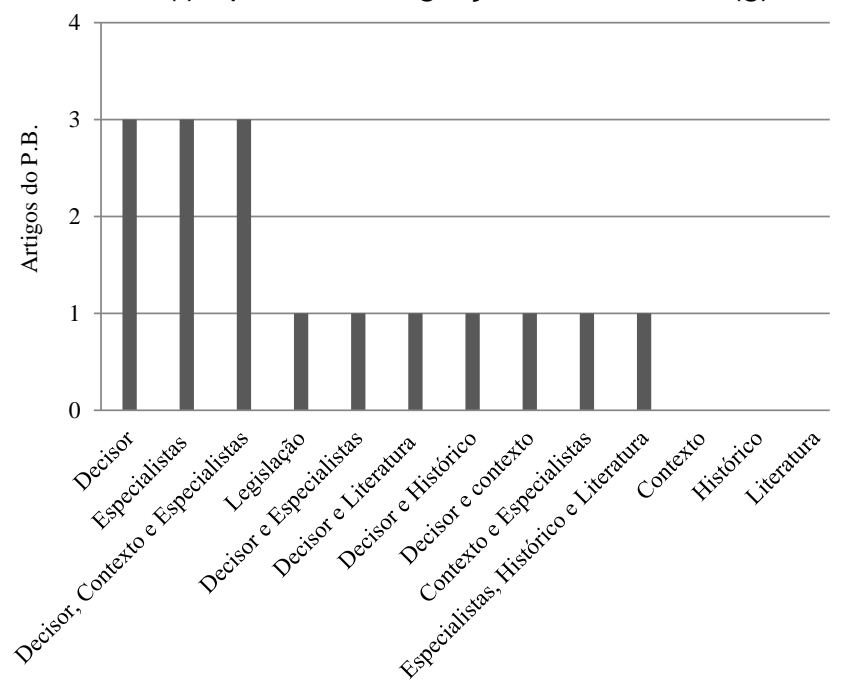

Base para construção do modelo

(a)

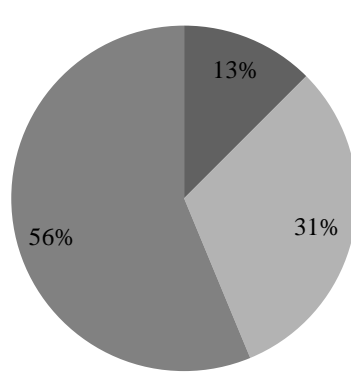

- Número de artigos que explicitam (identificam) o decisor no trabalho e o tem em conta para a construção integral do modelo

- Número de artigos que explicitam (identificam) o decisor no trabalho, mas não o tem em conta para a construção integral do modelo

- Número de artigos que não explicitam (identificam) o decisor no trabalho

(c)

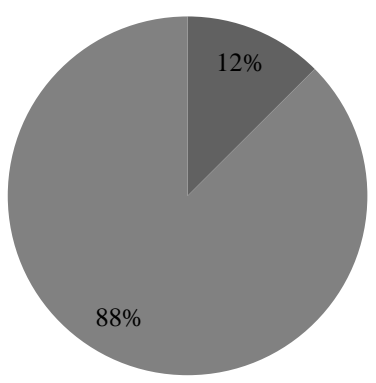

- Número de artigos em que o processo utilizado para identificar os objetivos está integralmente alicerçado nos valores do decisor

- Número de artigos em que o processo utilizado para identificar os objetivos está parcialmente alicerçado nos valores do decisor

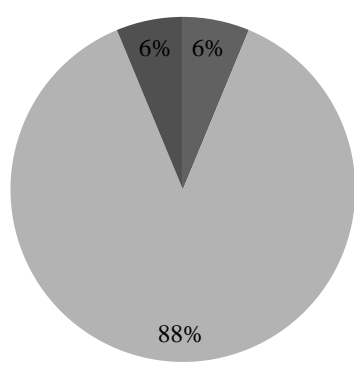

- O modelo foi construído e aplicado nc mesmo contexto

- O modelo foi construído em um ambiente, adaptado e aplicado em outro

- O modelo foi construído em um contexto e aplicado em outro

- O modelo foi construído em um ambiente e não foi aplicado

(b)

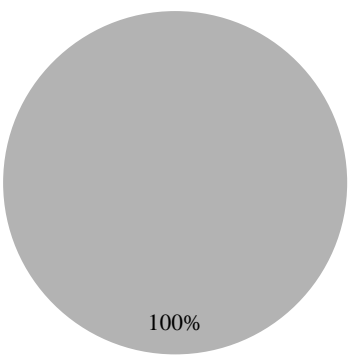

Número de artigos que desenvolve o modelo para um contexto físico e reconhece que o modelo é valido somente para este contexto físico

* Número de artigos que desenvolve o modelo para um contexto físico e o utiliza também em outros

(d)

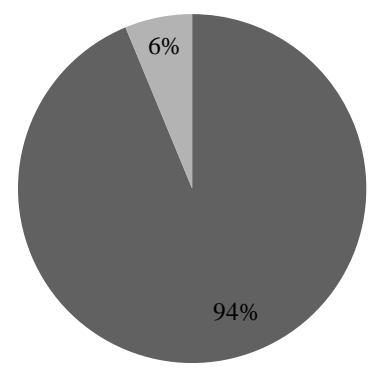

- Realiza a mensuração

ఐ Não realiza a mensuração

(e)

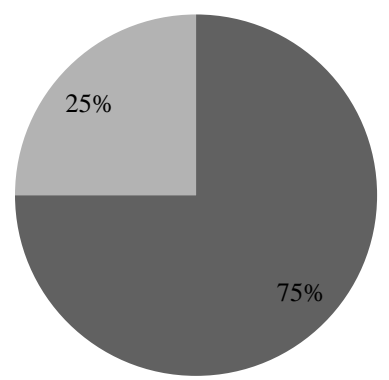

• Realiza a integração

não realiza a integração

(g) 
Sendo assim, foi possível verificar quanto à aplicação da lente abordagem, que a maior parte dos trabalhos apresentou a utilização de métodos já propostos na literatura, como por exemplo, método Analytic Network Process (MOLINOS et al., 2015; MOLINOS et al., 2014); Analytic Hierarchy Process (KALBAR et al., 2012; KALBAR et al., 2013); Data envelopment analysis (SALA et al., 2011); Técnica de simulação de Monte Carlo (FLORES et al., 2008). Outros autores apresentaram em seus trabalhos a junção de diferentes ferramentas com pequenas adaptações, que possibilitam a aplicação em diferentes contextos (ZENG et al., 2007; VASILOGLOU et al., 2009; RASHID et al., 2011; KARIMI et al., 2011; MOLINOS et al., 2012; KALBAR et al., 2012b; GARRIDO et al., 2014). Ao passo que apenas dois trabalhos apresentaram modelos desenvolvidos pelos autores (REFSGAARD, 2006; MAURER et al., 2012).

Foi observado que $31 \%$ dos trabalhos identificam o decisor no trabalho e destacam sua importância (Figura 4(c)), entretanto, não o tem em conta para a construção integral do modelo, sendo citado por estes trabalhos como agente fundamental na definição de pesos e julgamentos quanto aos critérios, mas não responsável pela total estruturação do modelo (ZENG et al., 2007; RASHID et al., 2011; KALBAR et al., 2012a; KARIMI et al., 2011; MOLINOS et al., 2015). Ao passo que, $56 \%$ não explicitam ou identificam o decisor no trabalho, mas apresentam outros fatores como sendo o agente de decisão, tais como: especialistas (GARRIDO et al., 2012; MAURER et al., 2012; KALBAR et al., 2013; MOLINOS et al., 2014), literatura (MOLINOS et al., 2012); e análise de séries temporais e/ou resultados passados (FLORES et al., 2008; KALBAR et al., 2012b; GARRIDO et al., 2014).

Considerando que o modelo, normalmente, tem por objetivo atender as necessidades ou a solução de um problema para um determinado decisor, fica evidente a necessidade de que esse seja levado em consideração na construção de todo o trabalho, entretanto, apenas dois dos artigos tem o decisor como agente fundamental para a construção integral do modelo (VASILOGLOU et al., 2009; REFSGAARD, 2006).

Deste modo, evidenciando a necessidade de estudos, especialmente quanto à tomada de decisão, que busquem compreender e ter suas escolhas fundamentada naquilo que o decisor julga importante, especialmente, no que diz respeito ao tratamento de efluentes, uma vez que a escolha de uma tecnologia de tratamento pode ser feita para longos períodos de tempo e esta deve atender e suprir as necessidades do decisor e suas expectativas. 
Quanto à lente singularidade em relação ao contexto (Figura 4(d)), foi observado que todos os trabalhos do portfólio bibliográfico desenvolveram seus modelos para um contexto físico e também utilizam ou permitem que esses sejam utilizados em outros contextos. Surge, portanto, nesta lente oportunidades de pesquisas que busquem contribuir com propostas direcionadas a contextos específicos considerando o maior número possível de variáveis intrínsecas a cada contexto avaliado, haja vista, que o contexto ao qual o modelo é aplicado é um agente influenciador direto no processo de tratamento de esgoto, ressaltando a importância e relevância de que este seja considerado (FERNANDES et al., 2001; SPERLING, 2014).

A lente referente aos valores do decisor (Figura $4(\mathrm{e})$ ) demonstrou que $88 \%$ dos trabalhos não tem em conta os valores do decisor quanto a identificação dos objetivos, ou não explicitam isso em seus trabalhos, ao passo que, aproximadamente $12 \%$ tem seus objetivos integralmente alicerçado nos valores do decisor. Emerge assim, de acordo com os resultados observados nas lentes singularidade quanto às características e valores do decisor, mais uma vez, há oportunidade de pesquisas que apresentem o enfoque de acordo com as percepções e necessidades do decisor, de modo que estes sejam considerados prioritariamente na construção e aplicação do modelo e ferramentas de apoio à decisão, no âmbito do tratamento de esgotos sanitários.

A lente quanto ao reconhecimento das limitações do decisor, de acordo com Ensslin et al. (2014) tem por objetivo compreender o processo apresentado pelo autor no artigo do portfólio bibliográfico com relação ao reconhecimento dos limites do conhecimento e entendimento do decisor. Assim sendo, não foi observado em nenhum trabalho do portifólio bibliográfico, caracteristicas como a utilização de critérios e/ou a operacionalização da avaliação da participação dos decisores, de maneira a considerar possiveis limitações referentes ao decisor.

Segundo com Ensslin et al. (2014) a mensuração tem por objetivo principal a avaliação da construção dos indicadores de desempenho, sendo assim, foi observado nos trabalhos do portfólio bibliográfico, conforme Figura 4(f), que 94\% dos trabalhos, ou seja 15 deles, realizaram e apresentaram de modo claro o processo utilizado de mensuração e avaliação dos indicadores também chamado pelos autores de critérios, ao passo, que apenas um trabalho não realizou a mensuração (SALA et al., 2011).

Segundo Ensslin et al. (2014) a integração pode ser definida como o processo para determinação do desempenho global por meio da definição de taxas de compensação para cada critério da avaliação. Conforme apresentado na Figura 4(g), com relação à lente 
integração dos critérios notou-se que $75 \%$ dos trabalhos realizaram a integração destes, ou seja, definiu taxas de compensação para cada critério definido na mensuração. O que já era esperado, considerando o elevado número de trabalhos que utilizaram ferramentas multicritério, as quais normalmente apresentam de maneira definida o processo de integração dos critérios.

\section{CONCLUSÕES}

Ao final do processo metodológico estruturado de busca da literatura foram selecionados 16 artigos com reconhecimento científico e alinhados com o tema. A cerca destes, foi possível conduzir o levantamento de diversas informações referentes à temática estuda, tais como: autores proeminentes, os periódicos relevantes para essa área de conhecimento, palavras-chave associadas ao tema, trabalho de maior reconhecimento cientifico, lacunas no conhecimento, entre outras informações relativas ao cenário internacional.

Dentre as análises referentes ao banco inicial de artigos alinhados a aplicação de ferramentas de decisão ao saneamento, foi possível observar um aumento considerável na produtividade durante o período coberto, de maneira especial a partir do ano de 2009. O que demonstrou uma crescente preocupação com as decisões envolvendo o saneamento, e por consequência a busca por ferramentas e métodos que auxiliem o avanço da área sanitária, de maneira a reduzir a subjetividade das decisões.

Quanto ao grau de relevância dos periódicos foi possível observar elevado fator de impacto para todos os periódicos que compunham o Portfólio bibliográfico. Fato este, que enfatiza a relevância do tema e suas contribuições para o meio acadêmico.

Grande parte dos artigos do portfólio bibliográfico e de suas referências foi publicado no periódico Journal of Environmental Management, ficando evidenciado, deste modo, grande destaque entre os demais periódicos em relação ao aceite do tema analisado.

A análise quanto ao reconhecimento científico dos artigos evidenciou que o autor de maior destaque entre os artigos das referências do portfólio bibliográfico foi Maria Molinos Senante. Quanto ao artigo de maior reconhecimento científico, elaborado por autores de destaque foi o artigo intitulado Optimization of wastewater treatment alternative selection by hierarchy grey relational analysis elaborado por Zeng et al. (2007), com elevado número de citação. 
Em relação aos resultados observados por meio da aplicação das lentes propostas por Ensslin et al. (2014) foi possível verificar uma série de oportunidades de pesquisas em diferentes aspectos da temática, principalmente quanto aos resultados das lentes singularidade em relação ao contexto, limites de conhecimento do decisor, gestão quando ao diagnóstico e aperfeiçoamento, notadamente sob os aspectos da perspectiva construtivista.

Tais lentes demonstraram que a construção dos modelos no cenário internacional, de modo geral, segue padrões possíveis de serem replicados em diferentes cenários, diante de diferentes aspectos e necessidades dos decisores. Surgindo assim a oportunidade de aplicação dos modelos em outros países, para avaliação do modelo e de seus resultados. Além disso, chama atenção para a discussão a respeito das peculiaridades dos cenários e suas intervenções na qualidade das respostas dos modelos.

Dos modelos de seleção analisados aproximadamente $88 \%$ foram construídos com base na literatura, especialistas e legislação, e só então aplicados a contextos específicos. Assim, evidenciando a necessidade de trabalhos com contextos decisórios menos generalistas que identifiquem as particularidades de cada cenário.

A ferramenta de intervenção utilizada para a busca da literatura demonstrou-se bastante eficiente, propiciando o conhecimento detalhado das características do conteúdo científico alinhado ao tema estudado.

\section{REFERÊNCIAS}

BORTOLUZZI, S. C.; ENSSLIN, S. R.; ENSSLIN, L; VALMORBIDA, S. M. L. A. Avaliação de desempenho em redes de pequenas e médias empresas: estado da arte para as delimitações postas pelo pesquisador. R. eletr. estrat. Neg, v. 4, n. 2, p. 202-222, 2011.

CAUCHICK, P. A. M. Metodologia da pesquisa em engenharia de produção e gestão de operações. Rio de Janeiro: Elsevier, 2012.

DUTRA, A.; RIPOLL-FELIU, V. M.; ENSSLIN, S. R.; ENSSLIN, L; ROGERIO, L.; GONÇALVES, P. Opportunities for research on evaluation of seaport performance: a systemic analysis from international literature. African J. Bus. Manag, v. 9, p. 704-717. 2015a.

DUTRA, A.; RIPOLL-FELIU, V. M.; FILLOL, A. G.; ENSSLIN, S. R.; ENSSLIN. L. The construction of knowledge from the scientific literature about the theme seaport performance evaluation. Int. J. Product. Perform. Manag. 64, p. 243-269. 2015b.

ENSSLIN, L.; GIFFHORN, E.; ENSSLIN, S.R.; PETRI, S. M.; VIANNA, W. B. Avaliação do Desempenho de Empresas Terceirizadas com o Uso da Metodologia Multicritério de Apoio à Decisão- Construtivista. Revista Pesquisa Operacional, v. 30, n. 1, p. 125-152, 2010. 
ENSSLIN, L; ENSSLIN, S. R.; PACHECO, G. C. Um Estudo Sobre Segurança em Estádios de Futebol Baseado na Análise da Literatura Internacional; Perspectivas em Ciências da Informação; v.17, n. 2, p. 71-91, 2012.

ENSSLIN, L; ENSSLIN, S. R.; SOUZA, M. V. Gerenciamento de portfólio de produtos na indústria: estado da arte. Revista Produção Online, v.14, n. 3, p. 790-821, 2014.

ENSSLIN, S. R.; ENSSLIN, L.; ADEMAR, L. D. S. M.; RIPOLL-FELIU, D. V. M. Research opportunities in performance measurement in public utilities regulation. Int. J. Product. Perform. Manag, v.64, n.7 p. 994- 1016. 2015.

FloRES, A., X.; RODRíGUEZ, R., I.; SIN, G.; GERNAEY, K. V. Multi-criteria evaluation of wastewater treatment plant control strategies under uncertainty. Water Res. v. 42, p. 4485-97. 2008.

GARRIDO, B., M. Including the environmental criteria when selecting a wastewater treatment plant. Environ. Model. Softw. v. 56, 74-82; 2014.

GARRIDO, B., M.; REIF, R.; HERNÁNDEZ, F.; POCH, M. Implementation of a knowledge-based methodology in a decision support system for the design of suitable wastewater treatment process flow diagrams. J. Environ. Manage. V. 112, 384-391, 2012.

GOOGLE SCHOLAR. Disponível em: http://scholar.google.com.br. (Acessado em novembro, 2015).

HAKANEN, J., MIETTINEN, K., \& SAHLSTEDT, K. Wastewater treatment: New insight provided by interactive multiobjective optimization. Decision Support Systems, v. 51, n. 2, p. 328-337, 2011.

HO, Y. S. Bibliometric analysis of biosorption technology in water treatment research from 1991 to 2004. Int J Environ Pollut, v. 34, p. 1-13, 2008.

HUNT, C. C. Modelo Multicritério de Apoio à Decisão Aplicado à Seleção de Sistema de Tratamento de Esgoto para Pequenos Municípios. Dissertação (Mestrado em Engenharia Ambiental) Universidade Federal do Rio de Janeiro. Rio de Janeiro, 2013.

KALBAR, P. P.; KARMAKAR, S.; ASOLEKAR, S. R. Selection of an appropriate wastewater treatment technology: A scenario-based multiple-attribute decision-making approach. J. Environ. Manage. 113, 158-169, 2012a.

KALBAR, P. P.; KARMAKAR, S.; ASOLEKAR, S. R. Technology assessment for wastewater treatment using multiple-attribute decision-making. Technol. Soc, v.34, p.295-302. 2012b.

KALBAR, P. P.; KARMAKAR, S.; ASOLEKAR, S. R. The influence of expert opinions on the selection of wastewater treatment alternatives: A group decision-making approach. J. Environ. Manage. v. 128, p. 844-851, 2013.

KARIMI, A. R.; MEHRDADI, N.; HASHEMIAN, S. J.; NABI BIDHENDI, G. R.; TAVAKKOLI MOGHADAM, R. Selection of wastewater treatment process based on the analytical hierarchy process and fuzzy analytical hierarchy process methods. Int. J. Environ. Sci. Technol. v, 8, p. 267280. 2011.

LACERDA, R. T. D. O.; ENSSLIN, L.; ENSSLIN, S. R. Research opportunities in strategic management field: a performance measurement approach. Int. J. Bus. Perform. Manag. v.15, p.158. 2014.

LIBÂNIO, M. Fundamentos de qualidade e tratamento de água. Campinas: Átomo, 2005. 444p. 
LI, J. F.; ZHANG, Y. H.; WANG, X. S.; HO, Y. S. Bibliometric Analysis of Atmospheric Simulation Trends in Meteorology and Atmospheric Science Journals. Croat Chem Acta, v. 82, p. 695-705, 2009.

LIMA, T. C. S.; MIOTO, R. C. T. Procedimentos metodológicos na construção do conhecimento científico: a pesquisa bibliográfica. Rev. Katál. Florianópolis, v. 10 n. esp. p. 37-45. 2007.

MAURER, M.; BUFARDI, A.; TILLEY, E.; ZURBRÜGG, C.; TRUFFER, B. A compatibility-based procedure designed to generate potential sanitation system alternatives. J. Environ. Manage. 104, 51-61, 2012.

METCALF; EDDY. Wastewater Engineering: treatment, disposal, reuse. 4. ed. New York: McGraw - Hill Book, 2003.

MOLINOS, S., M.; GÓMEZ, T.; CABALLERO, R.; HERNÁNDEZ-SANCHO, F.; SALA-GARRIDO, R. Assessment of wastewater treatment alternatives for small communities: An analytic network process approach. Sci. Total Environ. 532, 676-687, 2015.

MOLINOS, S., M.; GÓMEZ, T.; GARRIDO-BASERBA, M.; CABALLERO, R.; SALA, G., R. Assessing the sustainability of small wastewater treatment systems: a composite indicator approach. Sci. Total Environ. p.497-498, v.17, 2014.

MOLINOS, S., M.; GARRIDO, B., M.; REIF, R.; HERNÁNDEZ, F.; POCH, M. Assessment of wastewater treatment plant design for small communities: Environmental and economic aspects.

Sci. Total Environ, v. 427-428, p. 11-18, 2012.

RASHID, M. M.; HAYES, D. F. Needs-based sewerage prioritization: Alternative to conventional cost-benefit analysis. J. Environ. Manage, v. 92, p. 2427-2440; 2011.

REFSGAARD, K. Process-guided multicriteria analysis in wastewater planning. Environ. Plan. C Gov. Policy, v. 24, p. 191-213, 2006.

SAMPIERI, R. H.; COLLADO, C. F.; LUCIO, P. B. Metodologia de pesquisa. 3. ed. São Paulo: McGraw-Hill, 2006.

SALA, G., R.; MOLINOS-SENANTE, M.; HERNÁNDEZ-SANCHO, F. Comparing the efficiency of wastewater treatment technologies through a DEA metafrontier model. Chem. Eng. J. v. 173, p. 766-772. 2011.

VASILOGLOU, V.; LOKKAS, F.; GRAVANIS, G. New tool for wastewater treatment units location. Desalination, v. 248, p.1039-1048, 2009.

SPERLING, M. Introdução à qualidade da água e ao tratamento de esgoto, princípio do tratamento biológico de águas residuárias. 4. Ed. Belo Horizonte, p. 470, 2014.

SPERLING, M., CHERNICHARO, C. A. L. Urban wastewater treatment technologies and implementation of discharge standards in developing countries. Urban water. v. 4. p. 105-114. 2002.

ZHANG, L.; ZHOU, P.; NEWTON C., S.; JIAN, X.; FANG, D.; ZHOU, D.; ZHANG, L. Evaluating clean energy alternatives for Jiangsu, China: An improved multi-criteria decision making method. Energy xxx. v. 4, p. 124-136, 2015.

ZENG, G., JIANG, R.; HUANG, G.; XU, M.; LI, J. Optimization of wastewater treatment alternative selection by hierarchy grey relational analysis. J. Environ. Manage. v. 82, p. 250-259. 2007. 
ZHI, W.; GUODONG, J. Constructed wetlands, 1991-2011: A review of research development, current trends, and future directions. Science of the Total Envirm, v. 441. P. 19-27, 2012. 